\title{
Magnetic Origin of Giant Magnetoelectricity in Doped Y-type Hexaferrite $\mathrm{Ba}_{0.5} \mathrm{Sr}_{1.5} \mathrm{Zn}_{2}\left(\mathrm{Fe}_{1-x} \mathrm{Al}_{x}\right)_{12} \mathrm{O}_{22}$
}

\author{
Woo-Suk Noh, ${ }^{1}$ Kyung-Tae Ko, ${ }^{1,2,3}$ Sae Hwan Chun, ${ }^{4}$ Kee Hoon Kim, ${ }^{4}$ Byeong-Gyu Park, ${ }^{5}$ \\ Jae-Young Kim, ${ }^{5}$ and Jae-Hoon Park ${ }^{1,2,6, *}$ \\ ${ }^{1} c_{-} C C M R$ and Department of Physics, Pohang University of Science and Technology, Pohang 790-784, Republic of Korea \\ ${ }^{2}$ Max Planck POSTECH Center for Complex Phase Materials, Pohang University of Science and Technology, \\ Pohang 790-784, Republic of Korea \\ ${ }^{3}$ Max Planck Institute for Chemical Physics in Solid, 01187 Dresden, Germany \\ ${ }^{4}$ FPRD, Department of Physics and Astronomy, Seoul National University, Seoul 151-747, Republic of Korea \\ ${ }^{5}$ Pohang Accelerator Laboratory, Pohang University of Science and Technology, Pohang 790-784, Republic of Korea \\ ${ }^{6}$ Division of Advanced Materials Science, Pohang University of Science and Technology, Pohang 790-784, Republic of Korea
}

(Received 8 December 2014; published 19 March 2015)

\begin{abstract}
We investigated site-specific magnetic behaviors of multiferroic $\mathrm{Ba}_{0.5} \mathrm{Sr}_{1.5} \mathrm{Zn}_{2}\left(\mathrm{Fe}_{1-x} \mathrm{Al}_{x}\right){ }_{12} \mathrm{O}_{22}$ using $\mathrm{Fe}$ $L_{2,3}$-edge $\mathrm{x}$-ray magnetic circular dichroism. The $\mathrm{Al}$ dopants mostly replace the $\mathrm{Fe}^{3+}$ ions at octahedral $\left(O_{h}\right)$ sites, which contribute unquenched angular momenta through off-centering displacements. This replacement greatly reduces the magnetic anisotropy energy to change the magnetic order from a helical to a heliconical type with enhanced magnetoelectric susceptibility $\left(\alpha_{\mathrm{ME}}\right)$. The tetrahedral $\left(T_{d}\right) \mathrm{Fe}$ sites exhibit magnetic hysteresis distinguishable from that of the $O_{h}$ sites, especially at low magnetic fields. These results provide essential clues for the heliconical order with a giant $\alpha_{\mathrm{ME}}$ and multibit memory effects in the Al-doped Y-type hexaferrite.
\end{abstract}

DOI: 10.1103/PhysRevLett.114.117603

Multiferroic materials, in which magnetism and ferroelectricity coexist with a cross coupling, the so-called magnetoelectric (ME) effect, have been intensively studied experimentally and theoretically over the past decade due to their potential technological applications as nextgeneration multibit memory devices [1-3]. The ME effect was demonstrated in various multiferroic materials such as chromates, manganites, ferrites, etc. [1-6], and spiral, cycloidal, or noncollinear helical magnetic orders were known to be essential for a large ME effect [7-11]. Multiferroicity is well explained by an inverse Dzyaloshinskii-Moriya interaction [12,13] or a spin-current model [14]. However, most of the materials were recognized to face the difficulty of application barriers due to the low ME response, low ME temperature, and/or the requirement of a high magnetic field. Recently, hexaferrites with several different types have attracted much attention as candidates for possible multiferroic application materials with a giant ME susceptibility $\left(\alpha_{\mathrm{ME}}\right)$ at a relatively high temperature [4,15-17], and even nonvolatile multistate behaviors were demonstrated at room temperature [18]. Particularly in Y-type hexaferrites $(\mathrm{Ba}, \mathrm{Sr})_{2} \mathrm{Zn}_{2} \mathrm{Fe}_{12} \mathrm{O}_{22}$, small $\mathrm{Al}$ doping greatly enhances $\alpha_{\mathrm{ME}}$ at a low magnetic field [16].

Y-type hexaferrites $\mathrm{Ba}_{0.5} \mathrm{Sr}_{1.5} \mathrm{Zn}_{2}\left(\mathrm{Fe}_{1-x} \mathrm{Al}_{x}\right)_{12} \mathrm{O}_{22}$ are crystallized in a stacked hexagonal structure at a $R \overline{3} \mathrm{~m}$ space group $[19,20]$ with spinel structured blocks consisting of octahedral $\left(O_{h}\right)$ and tetrahedral $\left(T_{d}\right) \mathrm{Fe}$ sites as shown in Fig. 1(a). The magnetic structure is
PACS numbers: 77.80.-e, 75.30.Gw, 75.60.Ej, 78.70.Dm

conventionally described in terms of alternative stacked $L$ and $S$ blocks with large and small magnetic moments, respectively $[4,20]$. The magnetic ordering temperatures were reported to be $337 \mathrm{~K}$ for $x=0.00$ and $263 \mathrm{~K}$ for $x=0.08$ [16]. The spin structures are schematically depicted in Fig. 1(b). For $x=0.00$, both $L$ and $S$ block spins are aligned in a helical order under an in-plane magnetic field $H \| a b(\sim 1 \mathrm{~T})$ [20]. The ferroelectricity was
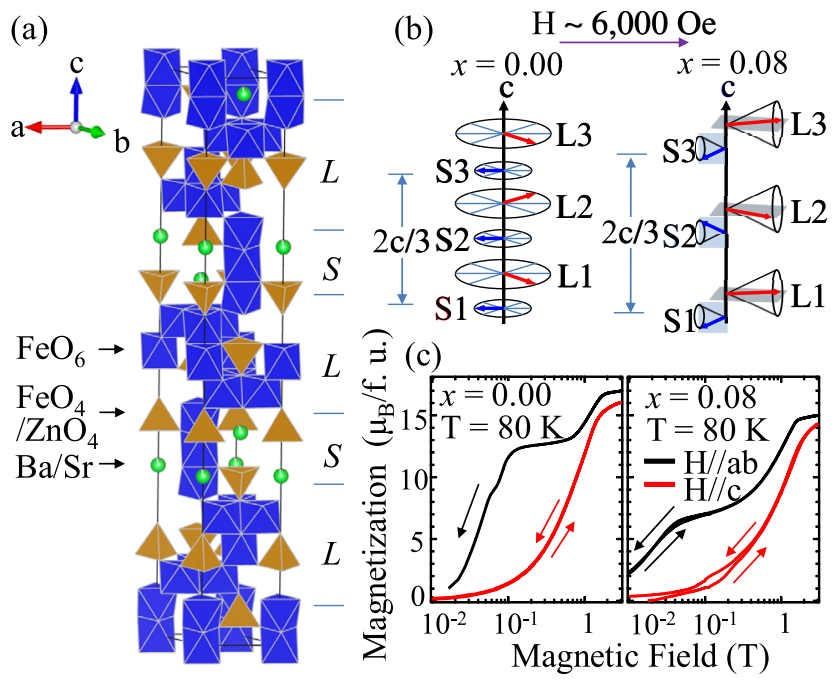

FIG. 1 (color online). (a) Crystal structure of Y-type hexaferrite. (b) Spin structures of $\mathrm{Ba}_{0.5} \mathrm{Sr}_{1.5} \mathrm{Zn}_{2}\left(\mathrm{Fe}_{1-x} \mathrm{Al}_{x}\right)_{12} \mathrm{O}_{22}$ for $x=0.00$ and 0.08 at $H=6000 \mathrm{Oe}$. (c) $M$ vs $H$ curves. 
proposed to be induced by an inversion symmetry breaking [4]. Meanwhile, for $x=0.08$, the spin order changes into a heliconical type with a certain $c$-direction magnetic component in the $S$ block under $H \| a b[16,21,22]$. The coneshaped spin blocks lead ferroelectricity with a greatly enhanced $\alpha_{\mathrm{ME}}$ through the spin-current mechanism katsura. The change in the spin structure was attributed to reduction of the in-plane magnetic anisotropy energy [15-17]. Indeed, the difference between the in-plane and out-ofplane magnetizations decreases with the doping in a 0.1-1 T region as shown in Fig. 1(c). Moreover, one can also recognize a certain in-plane remanent magnetization for $x=0.08$, which is essential for multibit memory device applications $[15,18]$.

In this Letter, we performed $\mathrm{x}$-ray magnetic circular dichroism (XMCD) measurements on the Y-type hexaferrites $\mathrm{Ba}_{0.5} \mathrm{Sr}_{1.5} \mathrm{Zn}_{2}\left(\mathrm{Fe}_{1-x} \mathrm{Al}_{x}\right)_{12} \mathrm{O}_{22} \quad(x=0.00,0.08)$ to explore a microscopic origin for the changes in the magnetic behavior with the doping and to find out a clue for the remanent magnetization. The results show unquenched orbital magnetic moments for both $x=0.00$ and 0.08 . The orbital moment $m_{o}$, which is mostly induced from off-centered octahedral $\left(O_{h}\right) \mathrm{Fe}$ sites, is reduced with the doping, resulting in a decrease of the in-plane magnetic anisotropy energy to change the spin structure with a largely enhanced $\alpha_{\mathrm{ME}}$. We also determined site-specific magnetic hystereses for the $O_{h}$ and $T_{d}$ Fe sites. The $T_{d}$ site hysteric behavior is distinguishable from that of the $O_{h}$ site with $\mathrm{Al}$ doping and originates a certain increase of remanent magnetization for the multistate behavior.

$\mathrm{Ba}_{0.5} \mathrm{Sr}_{1.5} \mathrm{Zn}_{2}\left(\mathrm{Fe}_{1-x} \mathrm{Al}_{x}\right)_{12} \mathrm{O}_{22} \quad(x=0.00,0.08)$ single crystals were grown by a flux method. Samples were cleaved in situ in a vacuum better than $5 \times 10^{-10}$ Torr. XMCD measurements were performed at the $2 A$ soft $\mathrm{x}$-ray elliptically polarized undulator beam line in Pohang Light Source. The results were obtained at $80 \mathrm{~K}$ with $\sim 95 \%$ circularly polarized light. A $0.5 \mathrm{~T}$ electromagnet was used for magnetization switching, the photon incident angle is $22.5^{\circ}$ off from the $H$ direction $(H \| c$ or $H \| a b)$, and XMCD spectra were collected in the total electron yield mode. The magnetization data were obtained by using a Quantum Design physical property measurement system.

To explore magnetic anisotropy of the system, we performed Fe $L_{2,3}$-edge XMCD measurements. The net spin moment direction was flipped to be parallel $\left(\rho_{+}\right)$ and antiparallel $\left(\rho_{-}\right)$to the photon helicity vector at each data point. The spectra were averaged over right and left helicities to minimize artificial effects. Figures 2(a) and 2(b) present the results of the absorption spectra ( $\rho_{+}$and $\left.\rho_{-}\right)$, dichroisms $\left(\Delta \rho=\rho_{+}-\rho_{-}\right)$, and integrations $\left[\int(\Delta \rho) d E\right]$ of the undoped $(x=0.00)$ and Al-doped $(x=0.08)$ samples, respectively. The degree of circular polarization and the geometry factor were taken into account in the spectra. The spectra are roughly divided into the $L_{3}\left(2 p_{3 / 2}\right)$ and $L_{2}\left(2 p_{1 / 2}\right)$ regions. The integrations
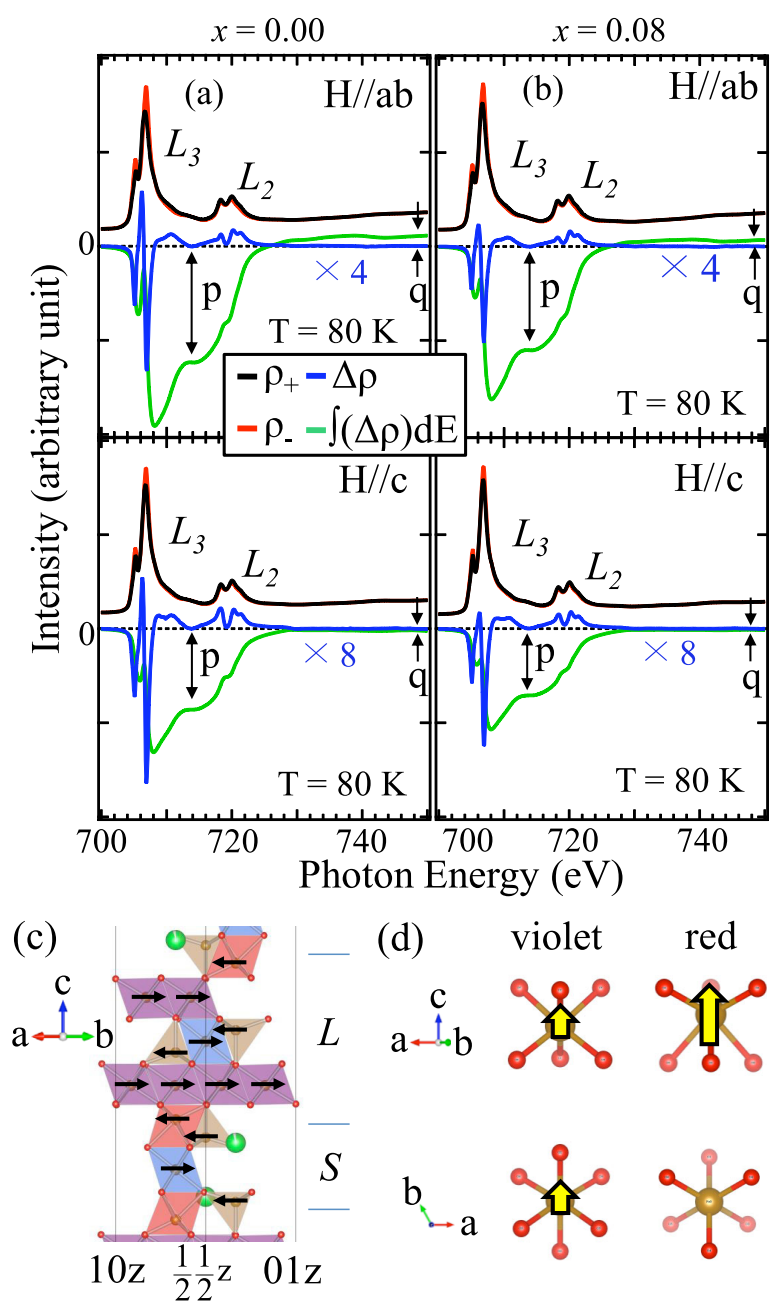

FIG. 2 (color online). Fe $L$-edge XMCD results of Y-type hexaferrite $\mathrm{Ba}_{0.5} \mathrm{Sr}_{1.5} \mathrm{Zn}_{2}\left(\mathrm{Fe}_{1-x} \mathrm{Al}_{x}\right)_{12} \mathrm{O}_{22}$ at $T=80 \mathrm{~K}, H=$ 6000 Oe for (a) $x=0.00$ and (b) $x=0.08$ under $H \| a b$ (top) and $H \| c$ (bottom). (c) Spin configurations of $\mathrm{Fe}^{3+}$ polyhedra under a saturation in-plane $H$ field. (d) Off-centering displacements of $\mathrm{Fe}^{3+}$ in two different $O_{h}$ sites.

over $L_{3}$ and entire $L_{2,3}$ regions are presented with $p$ and $q$, which are roughly proportional to the spin and orbital magnetic moments $m_{s}$ and $m_{o}$, respectively [23]. The obtained $p$ values are consistent with the magnetizations at $80 \mathrm{~K}$ under $H=6000 \mathrm{Oe}$. Using the sum rule [23], we estimated the orbital to spin moment ratio $m_{o} / m_{s}=$ $2 q /(9 p-6 q)=-0.018$ for $H \| a b\left(H_{a b}\right)$ and $m_{o} / m_{s}=$ +0.0046 for $H \| c\left(H_{c}\right)$. As the spins are fully aligned (eight up- and four down-spin $\mathrm{Fe}^{3+}$ ions) [4,20], the ionic magnetic moment becomes $20 \mu_{B} /$ f.u. $(\sim 10 \%$ larger than the saturated moment $M_{S}$ at $10 \mathrm{~K}$ ), and $m_{o}=-0.38 \mu_{B} /$ f.u. for $H_{a b}$ and $m_{o}=+0.09 \mu_{B} /$ f.u. for $H_{c}$, resulting in in-plane magnetic anisotropy. There exist only half-filled $\mathrm{Fe}^{3+}(L=0, S=5 / 2)$ ions, and off-centering displacements can be considered as the origin of the nonvanishing $m_{o}$ as discussed in $\mathrm{GaFeO}_{3}$ [24]. Indeed, one can find the off-centering distortions in the Y-type hexaferrite $[19,25]$. 
Figure 2(c) shows the arrangement of $(\mathrm{Fe}, \mathrm{Zn}) \mathrm{O}_{4}\left(T_{d}\right)$ and $\mathrm{FeO}_{6}\left(O_{h}\right)$ polyhedra in the undoped hexaferrite with four $T_{d}$ sites (brown) and ten $O_{h}$ sites per formula unit (f.u.). The $O_{h}$ sites are classified into three different groups presented with three different colors (two red, six violet, and two blue $O_{h}$ sites) in the figure. The $T_{d}$ sites are distributed with two $\mathrm{Zn}^{2+}$ and two $\mathrm{Fe}^{3+}$ ions, while all the $O_{h}$ sites are occupied by $\mathrm{Fe}^{3+}$ ions. The $L$ block contains two $T_{d}$ sites $\left(\sim 0.67 \mathrm{Fe}^{3+}\right)$ and nine $O_{h}$ sites (two reds, six violets, and one blue), and the $S$ block has two $T_{d}$ sites $\left(\sim 1.33 \mathrm{Fe}^{3+}\right)$ and one $O_{h}$ site (blue) $[20,26]$. The spin directions are presented with black arrows for $H_{a b}$. A large off-centering displacement occurs along the $c$ axis at the red $O_{h}$ site, while at the violet $O_{h}$ site a small off-centering displacement does both along the $c$ axis and in the $a b$ plane, as depicted with thick arrows in Fig. 2(d). The offcentering is negligible at the $T_{d}$ and blue $O_{h}$ sites. The shortest to average $\mathrm{Fe}-\mathrm{O}$ bond length ratio within an $\mathrm{FeO}_{6}$ octahedron is 0.93 for the red and 0.98 for the violet [25], indicating that the obtained in-plane $m_{o}=-0.38 \mu_{B} /$ f.u. mostly comes from the two red sites. The magnitude of $m_{o}$ is comparable to that of $\mathrm{GaFeO}_{3}$ with the ratio of 0.92 $[24,27]$. Considering that the orbital angular momentum is parallel to the spin momentum in an $\mathrm{Fe}^{3+}$ [24], its negative sign results from the red site spin direction opposite to the net $M$ direction. Meanwhile, a smaller out-of-plane $m_{o}=+0.09 \mu_{B} /$ f.u. is mainly from the six violet sites with the spin direction parallel to the $M$ direction.

The Al doping largely reduces the in-plane magnetic anisotropy. For $x=0.08$, the ratio was obtained to be $m_{o} / m_{s}=-0.013$ under $H_{a b}=6000$ Oe and $m_{o} / m_{s}=$ +0.0044 under $H_{c}=6000$ Oe from the XMCD results. The Al dopants were known to mostly replace the $O_{h}$ sites [16]. By supposing that those are uniformly distributed only at the $O_{h}$ sites, the ionic magnetic moment becomes $17.1 \mu_{B} /$ f.u., and $m_{o}=-0.22 \mu_{B} /$ f.u. for $H_{a b}$ and $m_{o}=$ $+0.06 \mu_{B}$ /f.u. for $H_{c}$, resulting in about $40 \%$ reduction in the magnetic anisotropy energy. This reduction is responsible for the spin structure change from a helical to a heliconical order [Fig. 1(b)]. One may notice that the reduction in $m_{o}$ is rather large in comparison with the 0.08 doping rate, which increases up to $9.6 \%$ in average under the assumption of all $\mathrm{Al}$ dopants at the $O_{h}$ sites. This large reduction can be attributed to two factors: (i) a certain decrease of the off-centering displacement with a reduction of the $c$ lattice constant and (ii) a preference of the $\mathrm{Al}$ replacement for the red $O_{h}$ site [28]. A recent nuclear magnetic resonance study reported that the $\mathrm{Al}$ replacement rate at the off-centered $O_{h}$ site (red) is considerably larger than the rate at the other $O_{h}$ sites (violet and blue) [29].

Besides the variations in $m_{o} / m_{s}$, the MCD spectral line shape also varies with the $H$-field direction and the $\mathrm{Al}$ doping. To extract information on the magnetic ordered states, we analyzed the line shapes by using cluster model calculations with full atomic multiplets and configuration interactions, the so-called CI calculations [30]. The calculated MCD spectra for $\mathrm{FeO}_{6}\left(O_{h}\right)$ and $\mathrm{FeO}_{4}\left(T_{d}\right)$ are extracted by fitting the MCD spectrum of a ferrimagnet $\gamma-\mathrm{Fe}_{2} \mathrm{O}_{3}$ [31], in which the $O_{h}$ and $T_{d} \mathrm{Fe}^{3+}$ spins are antiferromagnetically ordered with a $5: 3$ ratio. In the calculations, the spin moments are estimated to be $4.61 \mu_{B}$ and $4.68 \mu_{B}$ for $\mathrm{Fe}^{3+}$ at the $O_{h}$ and $T_{d}$ sites, respectively, resulting in a maximum spin moment of $18.3 \mu_{B} /$ f.u. for $x=0.00$ [eight $O_{h}$ up spins and two $T_{d}$ and two $O_{h}$ down spins, i.e., a net spin moment of $6 \times m_{s}\left(O_{h}\right)$ minus $\left.2 \times m_{s}\left(T_{d}\right)\right]$. As the ratio $m_{o} / m_{s}=$ -0.018 is taken into account, the total moment becomes $18.0 \mu_{B} /$ f.u., which well agrees with the in-plane saturated moment $M_{S}=17.9 \mu_{B} /$ f.u. at $10 \mathrm{~K}$.

Figure 3 shows the calculated MCD spectra for the $L_{3}$ region, in comparison with the experimental ones, and the spin structures for $x=0.00$ and $x=0.08$ at $H_{a b}=$ $6000 \mathrm{Oe}$ and $H_{c}=6000 \mathrm{Oe}$. The variation in the line shape can be reproduced by tuning the $O_{h}$ to $T_{d}$ site MCD ratio. Although the spin alignment with the saturated $M$ has the $O_{h}$ to $T_{d}$ spin ratio of $3:-1$, the MCD ratio can be vary with the spin structure in the applied $H$ field smaller than the saturation field ( $>3 \mathrm{~T}$ ). In the $x=0.00$ case (undoped), the best fits were obtained with the ratios of $2.3:-1$ and 2.7: -1 for the $H_{a b}$ and $H_{c}$, respectively. For the $H_{a b}$, the spin structure has a helical order with an in-plane tilting angle $\phi$ of the $L$ block spin, the so-called intermediate-II phase $[4,20]$, and $\phi \simeq 78^{\circ}$ is estimated from the ratio 2.3: -1 and $M \simeq 13 \mu_{B} /$ f.u. ( $76 \%$ of $M_{S} \simeq 17 \mu_{B}$ at $80 \mathrm{~K})$. Meanwhile, $H_{c}$ tilts the spins to the $c$ direction and produces a conical order. Considering $M \simeq 0.48 M_{S}$ at $80 \mathrm{~K}$, the cone angles are estimated to be $\alpha \simeq 61^{\circ}$ for the $L$ block spin and the angle $\alpha^{\prime} \simeq 53^{\circ}$ for the $S$ block spin. In both spin structures by $H_{a b}$ and $H_{c}$, the angle between the $L$ and $S$ block spins is nearly maintained to be $\sim 140^{\circ}$.

In the $x=0.08$ case (Al doped), the spin structure becomes a heliconical order with a cone angle $\beta$ for $H_{a b}$. The MCD ratio is estimated to be $2.8:-1$, which gives a maximum spin moment $16.5 \mu_{B} /$ f.u. [a net moment of $5.6 \times m_{s}\left(O_{h}\right)$ minus $\left.2 \times m_{s}\left(T_{d}\right)\right]$. This value is consistent with the observed $M_{S}=16.2 \mu_{B} /$ f.u., which results in the maximum spin moment $16.4 \mu_{B}$ /f.u. with $m_{o} / m_{s}=-0.013$. The cone angle is estimated to be $\beta \simeq 48^{\circ}$ at $H_{a b}=6000 \mathrm{Oe}$ from $M=0.67 M_{S}$ at $80 \mathrm{~K}$. For $H_{c}=6000 \mathrm{Oe}$, the spin structure becomes the conical order again [see Fig. 3(d)]. The cone angles $\gamma \simeq 67^{\circ}$ and $\gamma^{\prime} \simeq 75^{\circ}$ were estimated from the $O_{h}$ to $T_{d}$ ratio of $3.5:-1$ and $M \simeq 0.41 M_{S}$ at $80 \mathrm{~K}$. The angle between the $L$ and $S$ block spins reduces to $\sim 120^{\circ}$.

The $L_{3}$ MCD line shape shown in Fig. 4(a) presents typical $O_{h}-T_{d} \mathrm{Fe}^{3+}$ ferrimagnet features $o$, $t$, and $o^{\prime}$ resulting from a ferrimagnetic order of a large $O_{h}$ site spin and a small $T_{d}$ site spin, as in $\gamma-\mathrm{Fe}_{2} \mathrm{O}_{3}$ [24]; the features $o$ and $o^{\prime}$ are due to the $O_{h}$ site up spin, and $t$ is due to the $T_{d}$ site down spin as can be seen in Fig. 3. Thus, we 

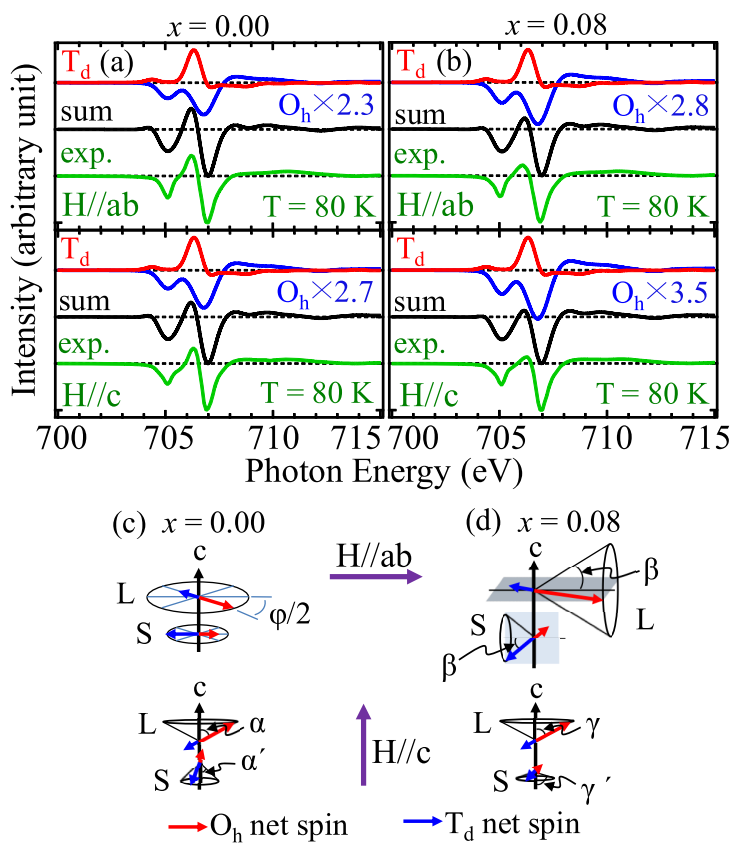

FIG. 3 (color online). Fe $L_{3}$-edge MCD spectra obtained from the CI calculations compared with the experimental ones for (a) $x=0.00$ and (b) $x=0.08$ in $\mathrm{Ba}_{0.5} \mathrm{Sr}_{1.5} \mathrm{Zn}_{2}\left(\mathrm{Fe}_{1-x} \mathrm{Al}_{x}\right){ }_{12} \mathrm{O}_{22}$ at $H \| a b$ and $H \| c$. Spin structures at $H=6000 \mathrm{Oe}$ and at $80 \mathrm{~K}$ for (c) $x=0.00$ and (d) $x=0.08$.

could obtain the site-specific magnetic hysteresis curves by monitoring the MCD peak intensity of each feature as a function of the $H$ field. Figures 4(b) and 4(c) show the $H \| a b$ and $H \| c$ magnetic hysteresis curves of the MCD features for $x=0.00$ and $x=0.08$, respectively. In the $x=0.00$ case, all three features display nearly identical hysteresis behaviors for both $H_{a b}$ and $H_{c}$, which coincide with one another with normalization factors corresponding to their different MCD intensities. Meanwhile, in the Aldoped $x=0.08$ case, the hystereses for the features $o$ and $o^{\prime}$, which are nearly identical to each other, are somewhat distinguishable from that for the feature $t$, especially in the low field region $-0.1 \mathrm{~T}<H<0.1 \mathrm{~T}$, indicating that the magnetic response of the $O_{h}$ site spin is different from that of the $T_{d}$ site spin.

The magnetic hystereses for the $O_{h}$ and $T_{d}$ site spins can be extracted by taking into account their MCD contributions at the specific photon energies of features $o, o^{\prime}$, and $t$ obtained from the CI calculation fits as presented in Fig. 3. Figures 4(d) and 4(e) show the normalized site-specific magnetic hystereses for $H \| a b$ and $H \| c$, respectively. Effective summations of these site-specific hystereses well reproduce the observed $M-H$ curves presented in Fig. 1(c) [31]. For $H \| a b$, the $T_{d}$ site has slightly larger values in the remanent ratio and coercive field, although its overall hysteresis behavior is similar to that of the $O_{h}$ site. On the other hand, the difference in the hysteresis behavior becomes more remarkable for $H \| c$ with much larger values in the ratio and field at the $T_{d}$ site. The nonmagnetic $\mathrm{Al}$ dopants, which (a) $\mathrm{T}=80 \mathrm{~K}, \mathrm{H} \sim 6,000 \mathrm{Oe}$

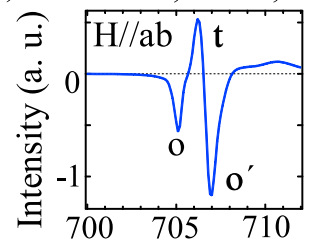

$$
\text { Photon Energy (eV) }
$$

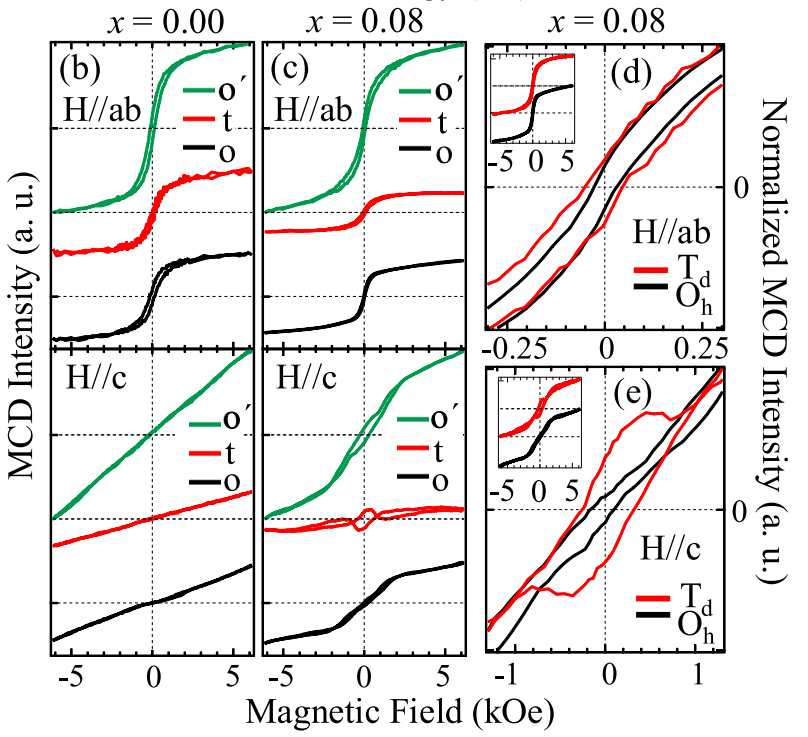

FIG. 4 (color online). (a) Fe $L_{3}$-edge MCD spectra of $\mathrm{Ba}_{0.5} \mathrm{Sr}_{1.5} \mathrm{Zn}_{2}\left(\mathrm{Fe}_{1-x} \mathrm{Al}_{x}\right){ }_{12} \mathrm{O}_{22} \quad(x=0.00)$. (b),(c) MCD intensities of the three features as a function of $H$ for (b) $x=0.00$ and (c) $x=0.08$. Here, the MCD intensity at the feature $t$ is flipped for convenience. (d) Normalized site-specific magnetic hystereses of the $O_{h}$ and $T_{d}$ sites determined from effective summations of the $H$-dependent MCD intensities.

mostly replace the $O_{h}$ sites, may loosen the magnetic exchange coupling of the neighboring $T_{d}$ site $\mathrm{Fe}$ spins to the $O_{h}$ site spins to make the magnetic behavior of the $T_{d}$ site separated from that of the $O_{h}$ site at the low magnetic field. This separated spin behavior may cause the change of the spin structure from helical to heliconical in the low field region in cooperation with the reduced in-plane magnetic anisotropy energy. As a result, the Al doping largely enhances the low field $\alpha_{\mathrm{ME}}$ and increases the remanent magnetization, which is essential for the multibit memory effects demonstrated in the multiferroic hexaferrites [18].

In summary, we reported detailed studies of magnetic origins for improved magnetoelectric effects in the doped Y-type hexaferrite $\mathrm{Ba}_{0.5} \mathrm{Sr}_{1.5} \mathrm{Zn}_{2}\left(\mathrm{Fe}_{1-x} \mathrm{Al}_{x}\right)_{12} \mathrm{O}_{22}$ using the Fe $L$-edge XMCD measurements. The Al dopants not only reduce the in-plane magnetic anisotropy energy to change the spin structure from a helical to a heliconical type but also loosen the magnetic coupling between the $T_{d}$ and $O_{h}$ sites resulting in distinguishable site-specific magnetic hysteresis behaviors. The results provide essential clues for the enhanced $\alpha_{\mathrm{ME}}$ and the multibit memory effects in the Al-doped Y-type hexaferrites. 
This work was supported by the National Creative Initiative (No. 2009-0081576 and No. 2010-0018300) and Max Planck POSTECH/KOREA Research Initiative (No. 2011-0031558) programs through NRF funded by MSIP of Korea. PAL is supported by POSTECH and MSIP of Korea.

* To whom all correspondence should be addressed. jhp@ postech.ac.kr

[1] T. Kimura, T. Goto, H. Shintani, K. Ishizaka, T. Arima, and Y. Tokura, Nature (London) 426, 55 (2003).

[2] B. B. Van Aken, J.-P. Rivera, H. Schmid, and M. Fiebig, Nature (London) 449, 702 (2007).

[3] S.-W. Cheong and M. Mostovoy, Nat. Mater. 6, 13 (2007).

[4] T. Kimura, G. Lawes, and A. P. Ramirez, Phys. Rev. Lett. 94, 137201 (2005).

[5] Y. Tokura and S. Seki, Adv. Mater. 22, 1554 (2010).

[6] I. A. Sergienko and E. Dagotto, Phys. Rev. B 73, 094434 (2006).

[7] M. Kenzelmann, A. Harris, S. Jonas, C. Broholm, J. Schefer, S. Kim, C. Zhang, S.-W. Cheong, O. Vajk, and J. Lynn, Phys. Rev. Lett. 95, 087206 (2005).

[8] G. Lawes et al., Phys. Rev. Lett. 95, 087205 (2005).

[9] K. Taniguchi, N. Abe, T. Takenobu, Y. Iwasa, and T. Arima, Phys. Rev. Lett. 97, 097203 (2006).

[10] T. Kimura, Annu. Rev. Mater. Res. 37, 387 (2007).

[11] H. Jang et al., Phys. Rev. Lett. 106, 047203 (2011).

[12] I. Dzyaloshinskii, J. Phys. Chem. Solids 4, 241 (1958).

[13] T. Moriya, Phys. Rev. 120, 91 (1960).

[14] H. Katsura, N. Nagaosa, and A. V. Balatsky, Phys. Rev. Lett. 95, 057205 (2005).

[15] S. Ishiwata, Y. Taguchi, H. Murakawa, Y. Onose, and Y. Tokura, Science 319, 1643 (2008).

[16] S. H. Chun et al., Phys. Rev. Lett. 104, 037204 (2010).

[17] S. Ishiwata, D. Okuyama, K. Kakurai, M. Nishi, Y. Taguchi, and Y. Tokura, Phys. Rev. B 81, 174418 (2010).
[18] S. H. Chun et al., Phys. Rev. Lett. 108, 177201 (2012).

[19] J. Smit and H. P. J. Wijn, in Ferrites (Philips' Technical Library, Eindhoven, 1959).

[20] N. Momozawa and Y. Yamaguchi, J. Phys. Soc. Jpn. 62, 1292 (1993).

[21] H. B. Lee, Y.-S. Song, J.-H. Chung, S. H. Chun, Y. S. Chai, K. H. Kim, M. Reehuis, K. Prokeš, and S. Mat'aš, Phys. Rev. B 83, 144425 (2011).

[22] H. Chang, H. B. Lee, Y.-S. Song, J.-H. Chung, S. A. Kim, I. H. Oh, M. Reehuis, and J. Schefer, Phys. Rev. B 85, 064402 (2012).

[23] C. T. Chen, Y. Idzerda, H.-J. Lin, N. Smith, G. Meigs, E. Chaban, G. Ho, E. Pellegrin, and F. Sette, Phys. Rev. Lett. 75, 152 (1995).

[24] J.-Y. Kim, T. Y. Koo, and J.-H. Park, Phys. Rev. Lett. 96, 047205 (2006).

[25] A. Collomb, J. Muller, J. C. Guitel, and J. M. Desvignes, J. Magn. Magn. Mater. 78, 77 (1989).

[26] S. Utsumi, D. Yoshiba, and N. Momozawa, J. Phys. Soc. Jpn. 76, 034704 (2007).

[27] E. F. Bertaut, G. Bassi, G. Buisson, J. Chappert, A. Delapalme, R. Pauthenet, H. P. Rebouillat, and R. Aleonard, J. Phys. (Paris) 27, 433 (1966).

[28] From the reduction value in the saturation magnetic moment with the $8 \%$ doping, the replacement rate at the red $O_{h}$ sites is estimated to be about $70 \%$ larger than the rates at other $O_{h}$ sites.

[29] S. Kwon, D. Y. Yoon, S. Lee, Y. S. Chai, S. H. Chun, and K. H. Kim, Phys. Rev. B 88, 064404 (2013).

[30] A. Tanaka and T. Jo, J. Phys. Soc. Jpn. 63, 2788 (1994).

[31] See Supplemental Material at http://link.aps.org/ supplemental/10.1103/PhysRevLett.114.117603 for additional information, which includes Refs. [24,32,33].

[32] S. Brice-Profeta, M. A. Arrio, E. Tronc, I. Letard, Ch. Cartier dit Moulin, and Ph. Sainctavit, Phys. Scr. T 115, 626 (2005).

[33] J. Chen, D. Huang, A. Tanaka, C. Chang, S. Chung, W. Wu, and C. Chen, Phys. Rev. B 69, 085107 (2004). 